CONTENTS (cont'd.)

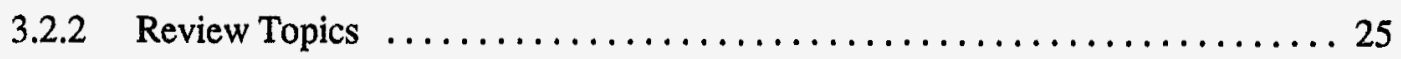

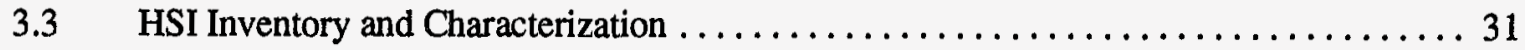

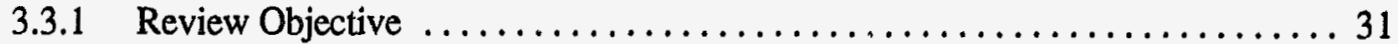

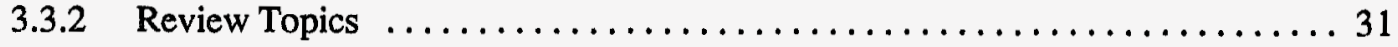

4 REVIEW OF HSI DESIGN VERIFICATION AND VALIDATION PHASE $\ldots \ldots \ldots \ldots \ldots . \ldots 35$

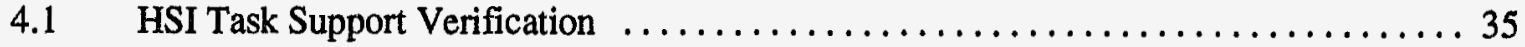

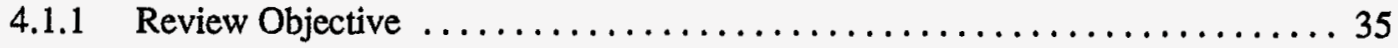

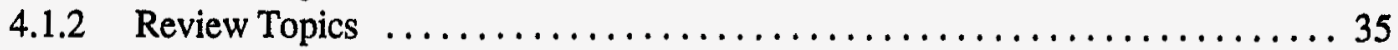

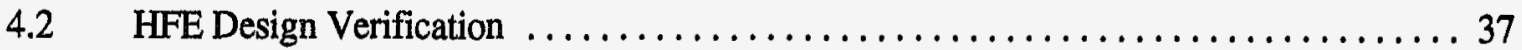

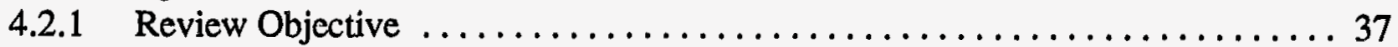

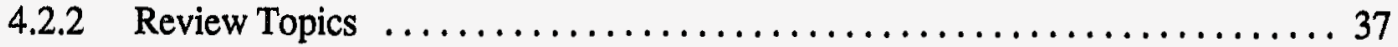

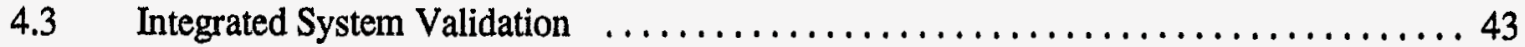

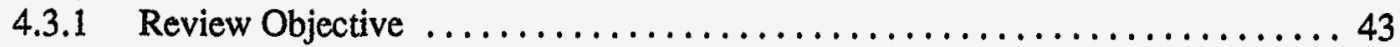

4.3 .2 Review Topics $\ldots \ldots \ldots \ldots \ldots \ldots \ldots \ldots \ldots \ldots \ldots \ldots \ldots \ldots, 43$

5 REVIEW OF HUMAN ENGINEERING DISCREPANCY RESOLUTION PHASE $\ldots \ldots \ldots \ldots 47$

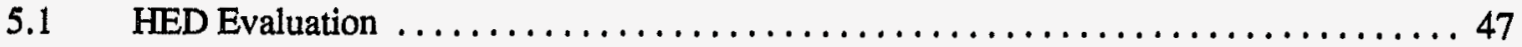

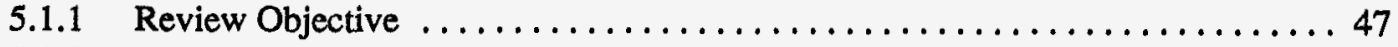

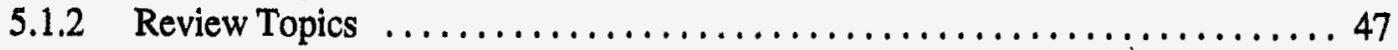

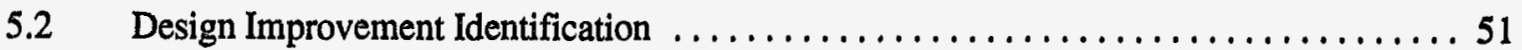

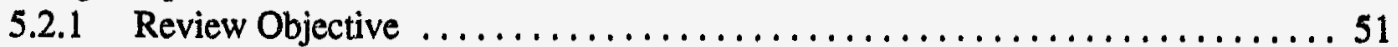

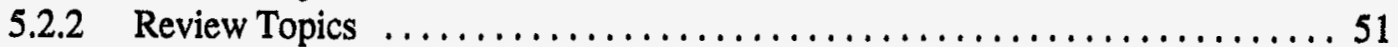

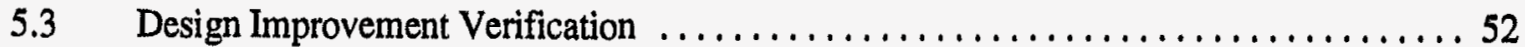

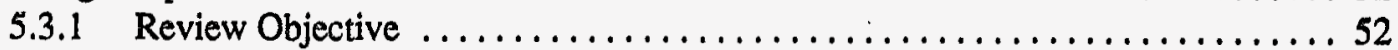

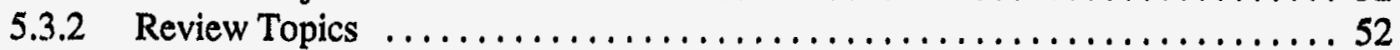

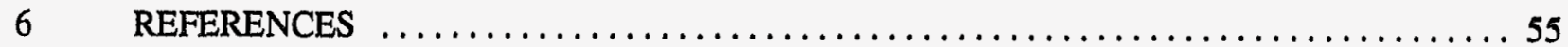




\section{CONTENTS (cont'd.)}

\section{Part 2: Guidelines for Human Factors Engineering Reviews}

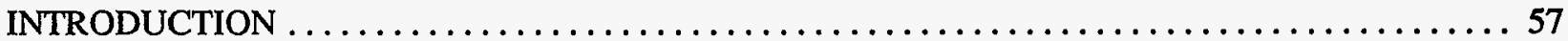

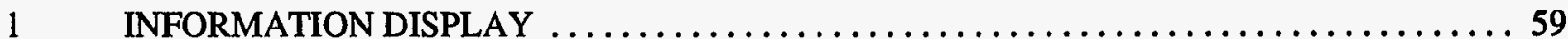

$1.1 \quad$ General Display Guidelines $\ldots \ldots \ldots \ldots \ldots \ldots \ldots \ldots \ldots \ldots \ldots \ldots \ldots \ldots \ldots$

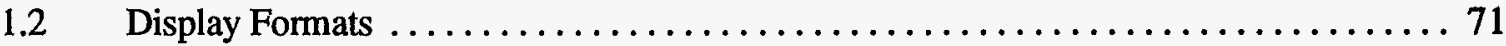

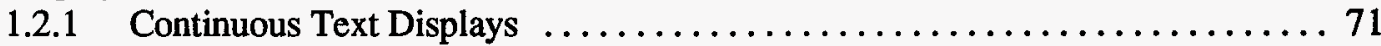

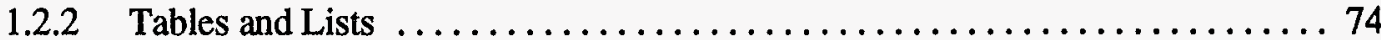

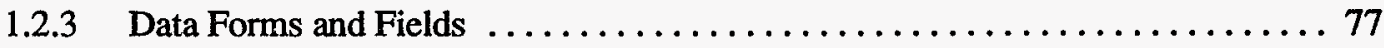

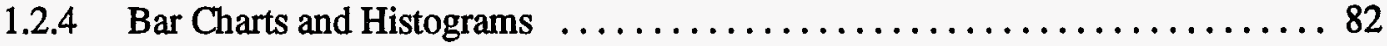

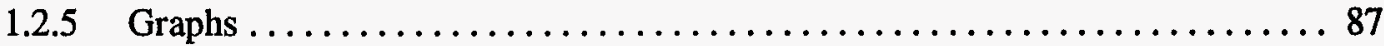

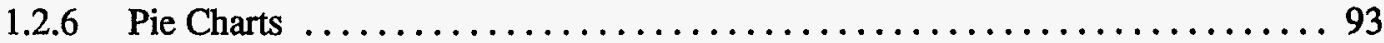

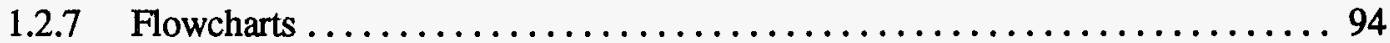

1.2.8 Mimics and Diagrams ................................... 95

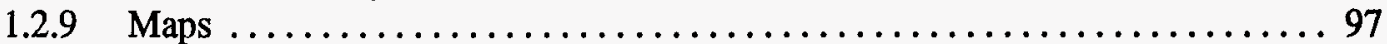

1.2.10 Graphic Instrument Panels ................................ 99

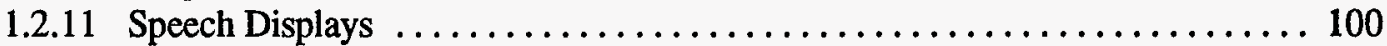

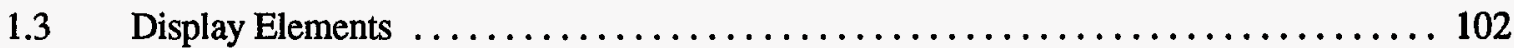

1.3.1 Alphanumeric Characters ................................ 102

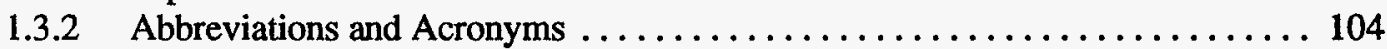

1.3 .3 Labels .......................................... 105

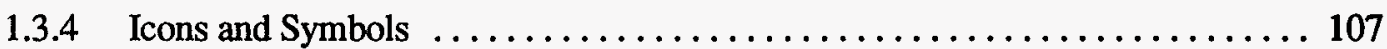

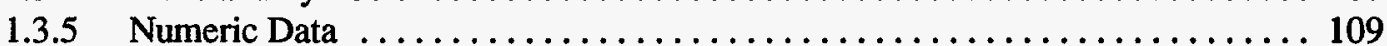

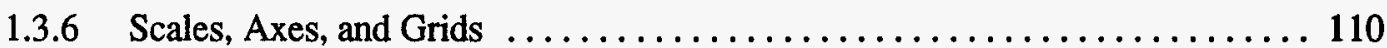

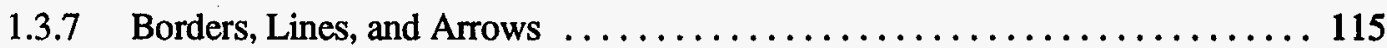

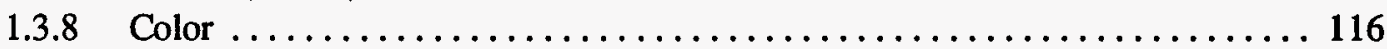

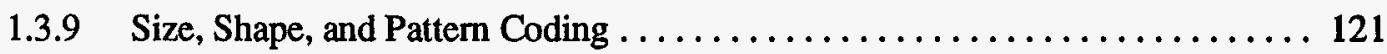

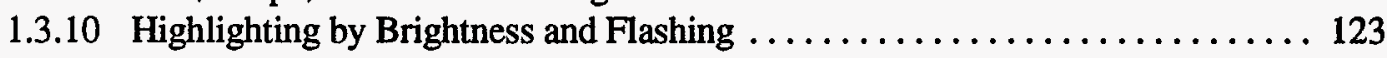

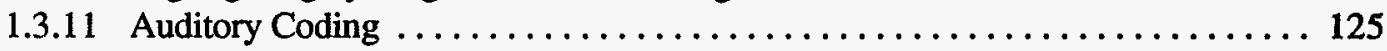

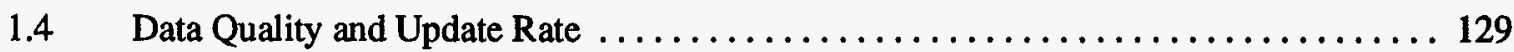

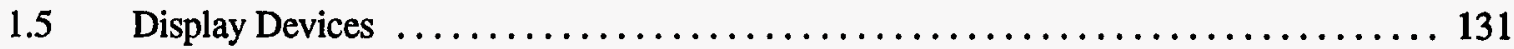

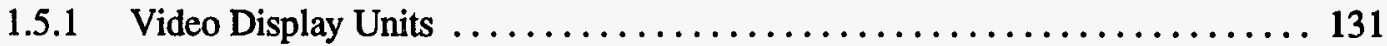

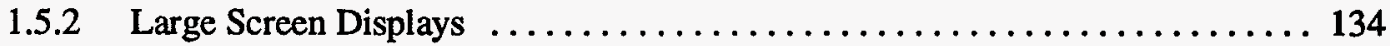

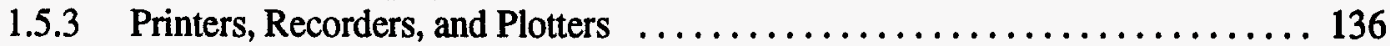

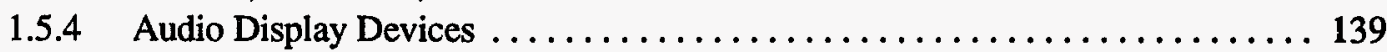

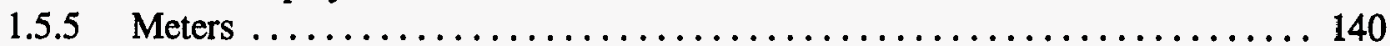




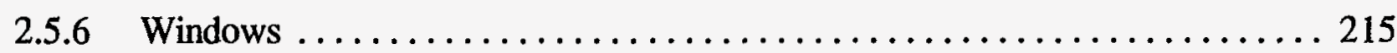

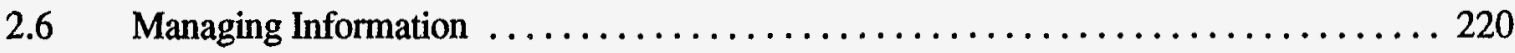

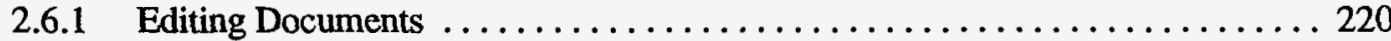

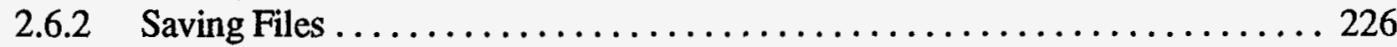

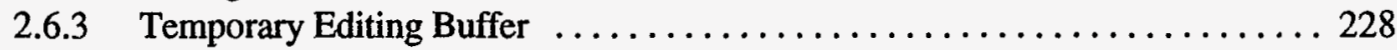

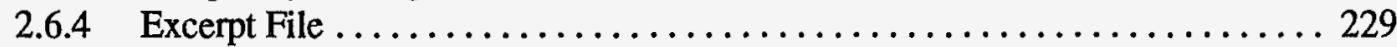

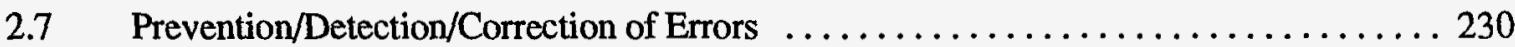

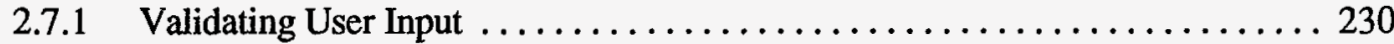

2.7.2 Correcting Information/Command Entries ..................... 232

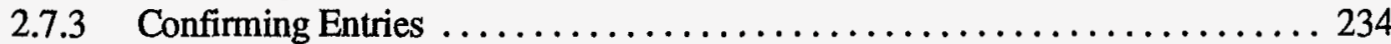

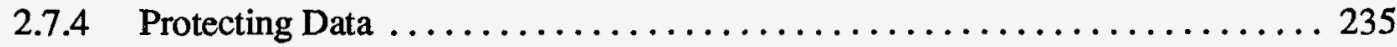

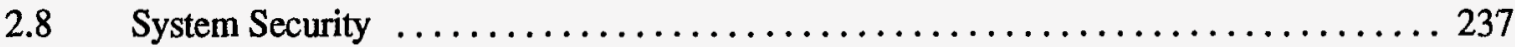

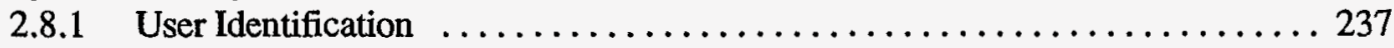

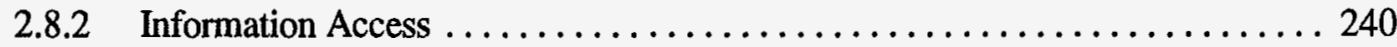

$3 \quad$ PROCESS CONTROL AND INPUT DEVICES $\ldots \ldots \ldots \ldots \ldots \ldots \ldots \ldots \ldots \ldots \ldots \ldots \ldots \ldots$

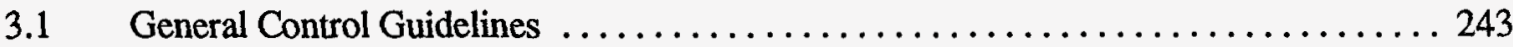

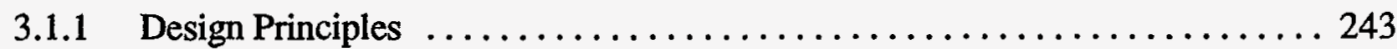

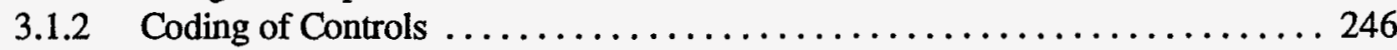

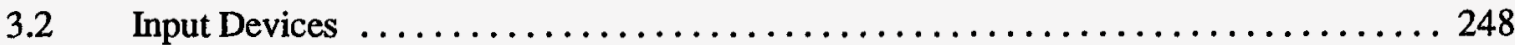

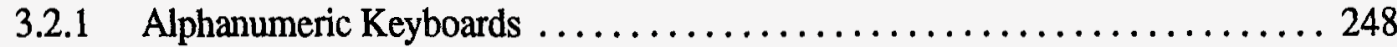

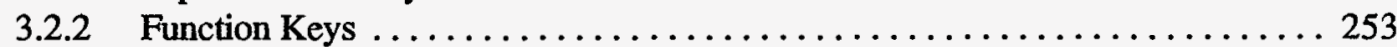

3.2.3 Trackballs, Joysticks, and Mice ............................ 255

3.2.4 Touch Screens, Light Pens, and Graphic Tablets . . . . . . . . . . . . 257

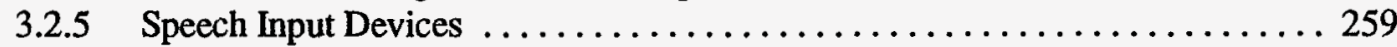

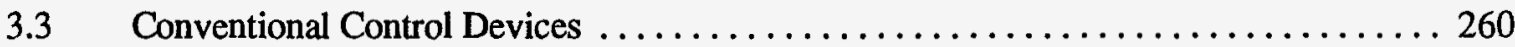

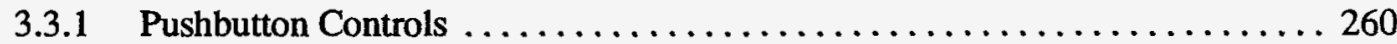

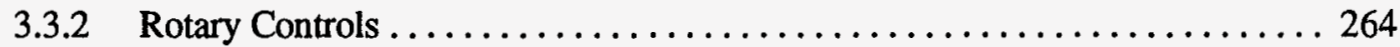

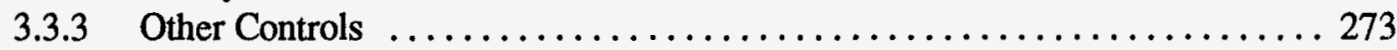

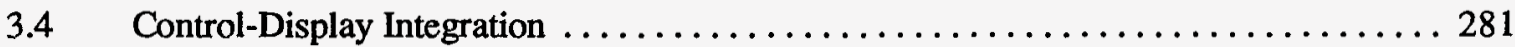

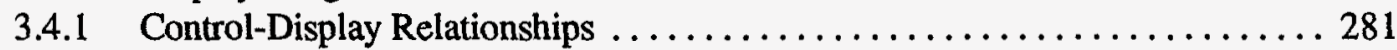

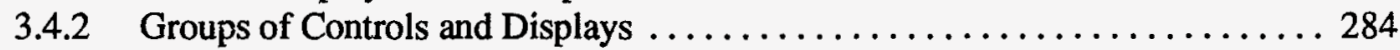

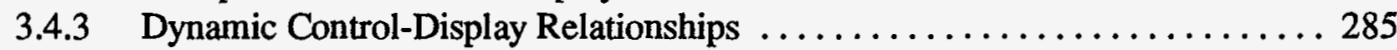




\section{CONTENTS (cont'd.)}

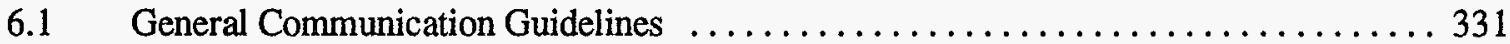

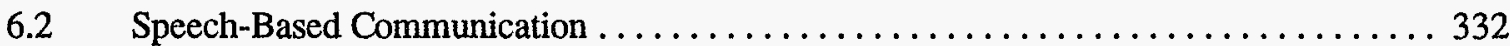

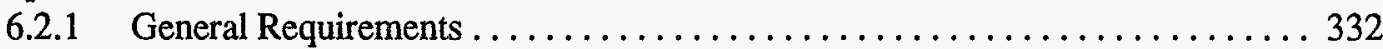

6.2.2 Conventional Telephone Systems ......................... 335

6.2 .3 Sound-Powered Telephone Systems ...................... 337

6.2.4 Portable Radio Transceivers . . . . . . . . . . . . . . . . . . . . . . . . . . . 338

6.2.5 Announcing Systems ................................ 339

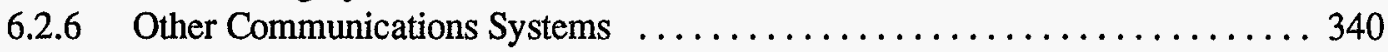

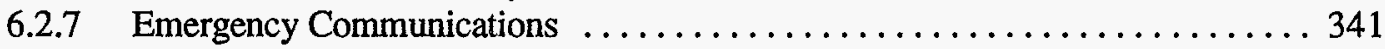

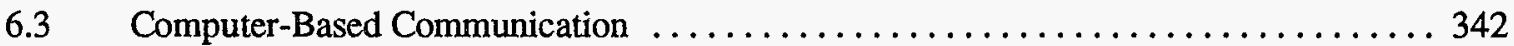

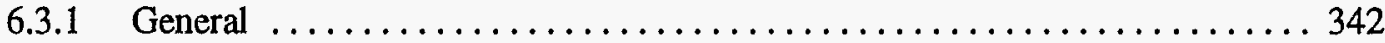

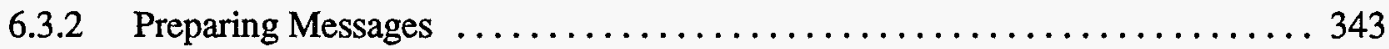

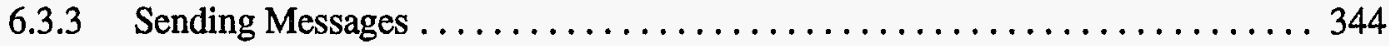

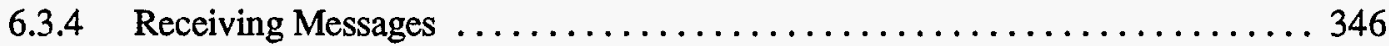

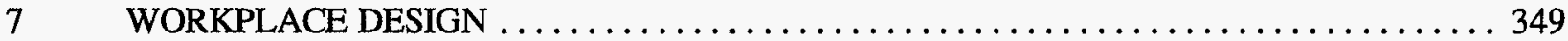

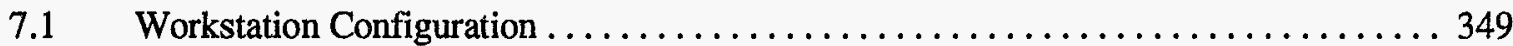

7.1.1 Stand-Up Console Dimensions ............................ 349

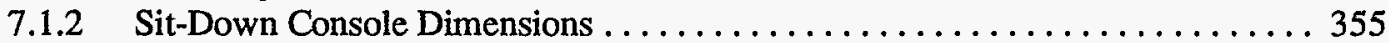

7.1.3 Sit-Stand Workstations . . . . . . . . . . . . . . . . . . . . . . . 361

7.1 .4 Vertical Panels .................................. 362

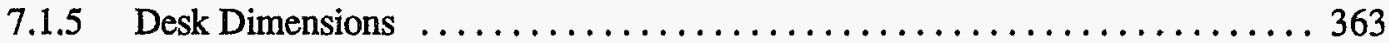

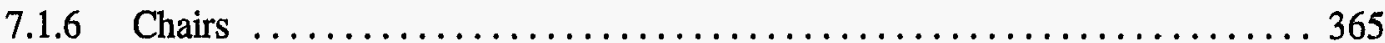

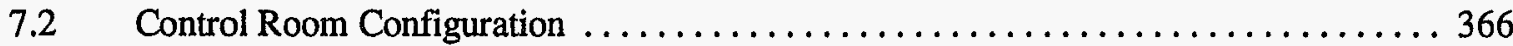

7.2.1 Accessibility of Instrumentation/Equipment $\ldots \ldots \ldots \ldots \ldots \ldots \ldots \ldots \ldots$

7.2 .2 Consistency of Manning with Equipment Layout $\ldots \ldots \ldots \ldots \ldots \ldots \ldots$

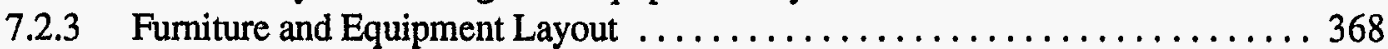

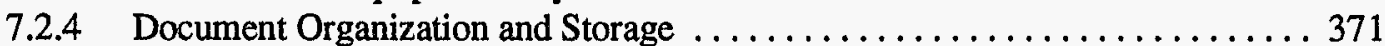

7.2.5 Spare Parts, Operating Expendables, and Tools $\ldots \ldots \ldots \ldots \ldots \ldots \ldots \ldots 2$

7.2 .6 Supervisor Access ...................................... 373

7.2 .7 Multiunit Control Rooms ................................ 374

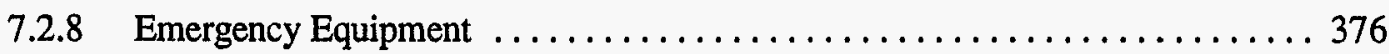




\section{CONTENTS (cont'd.)}

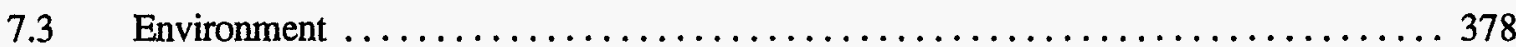

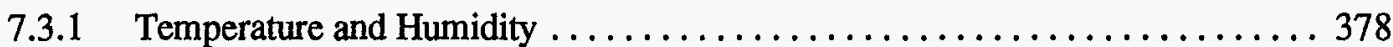

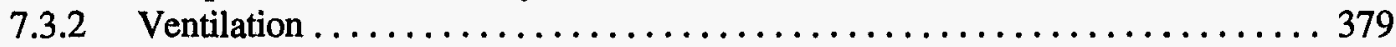

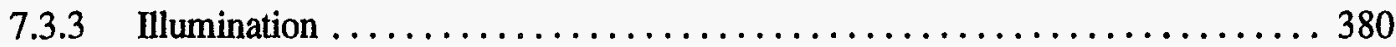

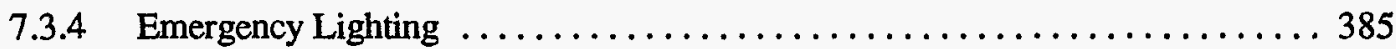

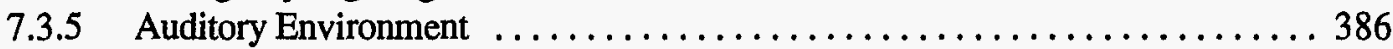

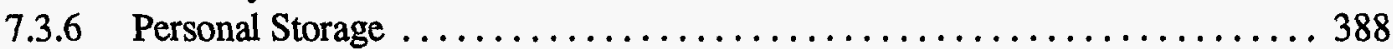

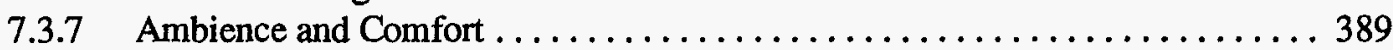

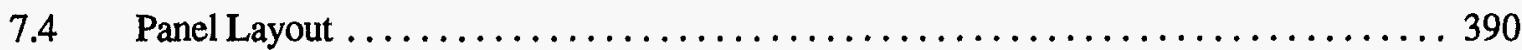

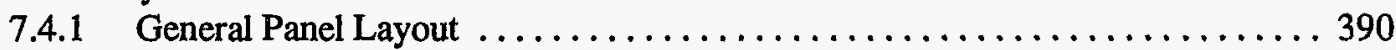

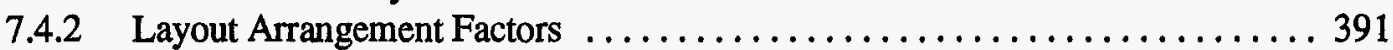

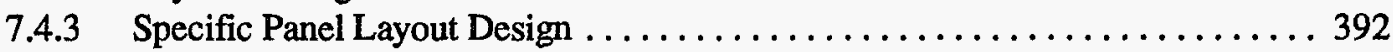

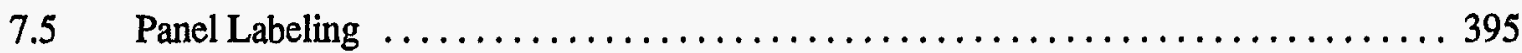

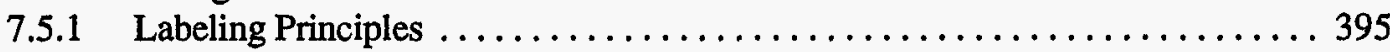

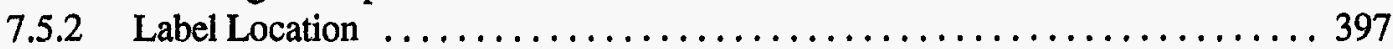

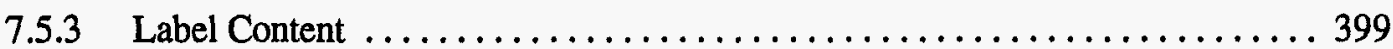

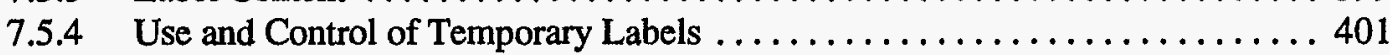

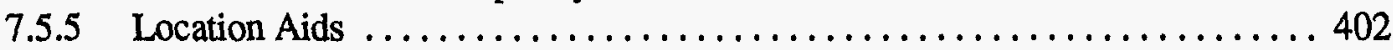

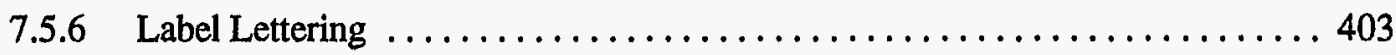

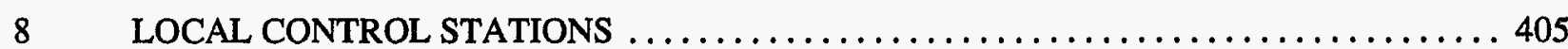

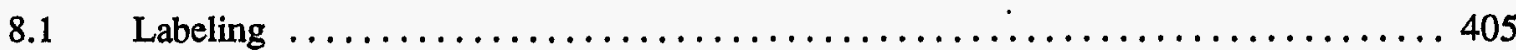

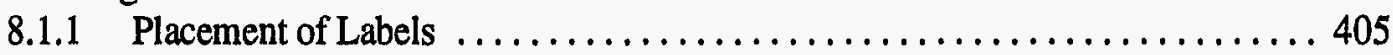

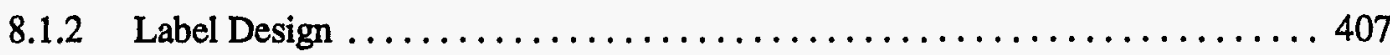

8.1.3 Label Content ................................... 409

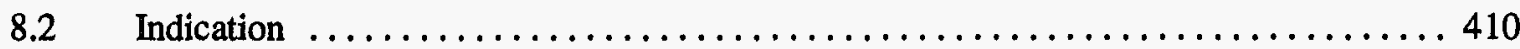

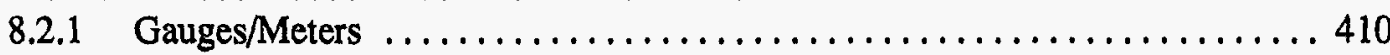

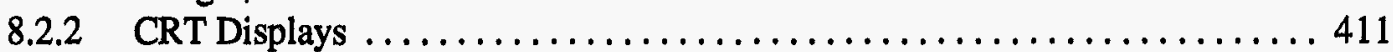

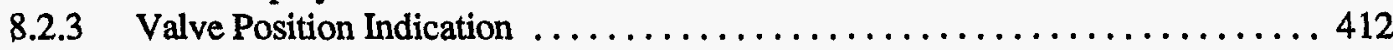

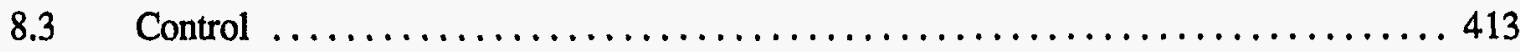

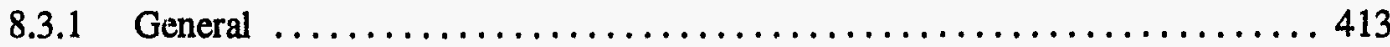

8.3.2 Manual Valves ....................................... 414

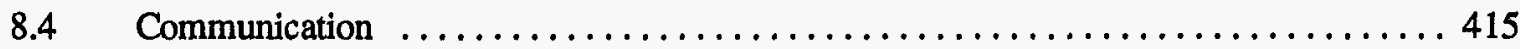

8.4 .1 Loudspeakers ................................... 415

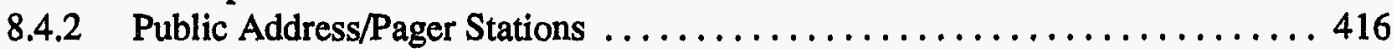

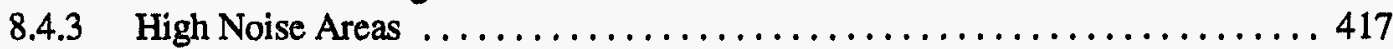




\section{EXECUTIVE SUMMARY}

\section{Introduction}

The importance of a well-designed human-system interface (HSI) to reliable human performance and nuclear power plant (NPP) safety is widely acknowledged. One of the first insights from studies of the Three Mile Island (TMI) accident was that errors resulting from human factors deficiencies in the control room (CR) were a significant contributing factor to NPP incidents and accidents. One significant outgrowth of the accident with respect to the human-system interface design was that the U.S. Nuclear Regulatory Commission (NRC) required all licensees and applicants for commercial NPP operating licenses to conduct detailed control room design reviews, including reviews of remote shutdown panels, to identify and correct human factors design deficiencies. Extensive guidelines published as NUREG-0700, "Guidelines for Control Room Design Reviews" (NRC, 1981b), were prepared in support of these reviews.

In further response to the Three Mile Island incident, the NRC staff focused on issues for which there were uncertainties in the scientific data needed to support regulation. One such issue was the introduction of advanced, computer-based HSI technology into control rooms and local control stations, a technology which was not used in TMI-era NPPs. In this document, the term "advanced" refers to HSI technologies such as touchscreen controls and large-screen, overview displays. Such technologies are advanced relative to the HSI designs implemented in most current plants. Advanced HSI designs are emerging in NPPs as a result of several factors: (1) replacement of existing CR HSIs with computer-based technologies when existing hardware is no longer supported by equipment vendors, (2) upgrades of CRs with new, computer-based monitoring and control systems, and (3) development of advanced control room (ACR) concepts as part of new (evolutionary and passive) reactor designs. These developments could have significant implications for plant safety in that they will affect the overall role (function) of personnel in the system, the amount of information available, the type of information available, the format of information presentation, the ways in which personnel interact with the system, and the requirements imparted upon personnel to understand and supervise an increasingly complex system.

To help assure that the incorporation of advanced technology into both new and existing CRs enhances the potential safety benefits and minimizes the potential negative effects on performance and plant safety, the NRC has programs in place to review the design and implementation of significant changes to existing CRs and to review the human factors engineering (HFE) aspects of $\mathrm{ACR}$ designs. However, the principal review guidance (NUREG-0700; NRC, 1981b) available to the NRC was developed more than ten years ago, well before these technological advances, and it was thus limited in its applicability to those technologies used in then existing CRs. Accordingly, the human factors guidance needed updating to serve as the basis for NRC review of advanced technologies.

This document, NUREG-0700, Rev. 1, provides guidance to the NRC staff for the following: (1) review of HSI design review submittals prepared by licensees or applicants for a license or design certification, and (2) performance of HSI reviews that could be undertaken as part of an inspection or other type of regulatory review involving HSI design or incidents involving human performance. It consists of two major parts. Part 1 describes those aspects of the HSI design review process that are important to the identification and resolution of human engineering discrepancies (HEDs). Guidance is provided that could be used by the staff to review an applicant's HSI design review process. Part 1 also could be used by the staff to guide the development of an HSI design 


\section{EXECUTIVE SUMMARY (cont'd.)}

review plan, e.g., as part of an inspection activity. Part 2, "Guidelines for Human Factors Engineering Reviews," provides detailed HFE guidelines which describe the specific review criteria for the assessment of the actual HSI design implementation. A summary of each of the parts of the document follows.

\section{Part 1: Review Methodology and Procedures}

The overall purpose of an HSI design review is to ensure that the HSI supports safe, efficient, and reliable personnel task performance. Thus, the objective of the staff's evaluation of an applicant's HSI design review is to ensure that the applicant has systematically identified and resolved HEDs that could adversely affect plant safety.

The scope of the HSIs addressed by NUREG-0700, Rev. 1, includes those used by plant personnel in the performance of their functions and tasks related to safe operation of the plant. Relevant HSIs include all alarms, displays, controls, job performance aids, workstation and workplace layouts, as well as environmental conditions such as lighting, noise, temperature, and humidity.

The staff's review ensures that the applicant has accomplished the following items:

Identified specifically HSI-related issues, adequately defined HSI design and performance requirements, and correctly characterized the HSI:

- Operating experience with human performance problems has been reviewed with regard to the specific system for which the HSI was designed, and the specific HSI technologies implemented.

- Function and task analyses which are needed to establish HSI design and performance requirements have been adequately completed and have addressed normal plant operation, design basis accident analyses of Chapter 15 of the plant's safety analysis report, and significant scenarios identified in the plant's probabilistic risk analysís.

- Identified and documented HEDs in the HSI design. Because no one method is likely to be sufficient, it may be necessary for the applicant to perform a series of analyses as described below:

- $\quad$ HSI Task Support Verification - An evaluation that verifies that the HSI supports all identified personnel task requirements as defined by task analyses. HEDs are identified for: (1) personnel task requirements that are not fully supported by the HSI, and (2) the presence of HSI components which are not needed to support a personnel task requirement, or which distract from task performance.

- $\quad$ HFE Design Verification - An evaluation that verifies that the HSI is designed and implemented to account for human capabilities and limitations. HEDs are identified if the design or implementation of the HSI is inconsistent with the HFE guidelines (see Part 2 of this document). 
(4) Technical Program Development

- The scope, procedures, products, and schedule should be defined for the following:

- Operating Experience Review

- Function and Task Analysis

- $\quad$ HSI Inventory and Characterization

- $\quad$ HSI Task Support Verification

- $\quad$ HFE Design Verification

- Integrated System Validation

- HED Evaluation

- Design Improvement Identification

- Design Improvement Verification

- $\quad$ The HFE requirements for designing upgrades or resolving HEDs should be identified, including the standards and specifications that are sources of HSI requirements.

- HFE facilities, equipment, tools, and techniques (such as laboratories, simulators, and rapid prototyping software) to be used in the HSI design review should be identified.

(5) HSI Design Review Reporting - Reports needed to complete the HSI design review should be identified, and their expected contents described. 
